\title{
The long non-coding RNA IncFOXO1 suppresses growth of human breast cancer cells through association with BAP1
}

\author{
JIE XI, JING FENG, QIAN LI, XIA LI and SAITIAN ZENG \\ Department of Gynecology, Cangzhou Central Hospital, Cangzhou, Heibei 061001, P.R. China
}

Received December 14, 2016; Accepted February 6, 2017

DOI: $10.3892 /$ ijo.2017.3933

\begin{abstract}
Breast cancer, one of the common cancers of women, is the leading cause of death among women below the age of 50 years in western countries. Long non-coding RNAs (lncRNAs) have been shown to be involved in diverse biological processes, both physical and pathological. However, to date, only a few lncRNAs have been functionally identified in breast cancer, and the overall pathophysiological contributions of lncRNAs to breast cancer remain largely unknown. In the present study, we identified a novel lncRNA termed lncFOXO1 through microarray screening. IncFOXO1 is significantly decreased in breast cancer tissues and cell lines and downregulation of 1 cFOXO1 expression associates with poorer overall survival. Functional assays demonstrated its suppressive role in breast cancer in vivo and in vitro. Mechanistically, lncFOXO1 suppressed the growth of breast cancer by increasing FOXO1 transcription. Moreover, we found that $1 \mathrm{ncFOXO1}$ associated with BRCA-1-associated protein 1 (BAP1) and regulates its binding and the level of mono-ubiquitinated H2A at K119 (ubH2AK119) at FOXO1 promoter.
\end{abstract}

\section{Introduction}

For several decades, the biological functions have been thought to depend on protein-coding genes. However, proteincoding genes accounts for only approximately $2 \%$ of the whole human genome (1). The majority is non-coding RNA (ncRNA) contains housekeeping ncRNAs and regulatory ncRNAs. Long non-coding RNAs (lncRNAs) are transcripts longer than 200 bp without any apparent protein-coding potential (2). Recent studies have demonstrated that lncRNAs are involved in diverse biological processes, both physical and pathological, and that they function as gene regulators at the transcriptional, post-transcriptional and post-translational level by directly interacting with DNA, RNA or proteins (3).

Correspondence to: $\mathrm{Dr} \mathrm{Jie} \mathrm{Xi}$, Department of Gynecology, Cangzhou Central Hospital, Hebei Medical University, Cangzhou, Heibei 061001, P.R. China

E-mail: xijiehmu@126.com

Key words: lncRNA, breast cancer, FOXO1, BAP1
Breast cancer, one of the common types of cancer for women, is the leading cause of death among women below the age of 50 years in western countries (4). Based on gene expression profiling, 4 different subtypes of breast cancer have been established, including luminal A, luminal B, HER2 and basallike (BLBC). Understanding the relationship between the clinical symptoms and the molecular changes in breast cancer is of great importance for developing new diagnosis and treatment strategies for breast cancer patients. Recent studies have indicated that dysregulation of lncRNAs is closely associated with human diseases including cancers $(5,6)$. For example, lncRNA focally amplified lncRNA on chromosome 1 (FAL1), whose copy number and expression are significantly correlated with poor prognosis of ovarian cancer patients. FAL1 directly interacts with the BMI1 and regulates its protein stability, and then modulates the transcription of several genes including CDKN1A (7). However, to date, only a few lncRNAs have been functionally identified in breast cancer, and the overall pathophysiological contributions of lncRNAs to breast cancer remain largely unknown.

In the present study, we identified a novel lncRNA termed lncFOXO1 through microarray screening, and demonstrated its suppressive role in breast cancer in vivo and in vitro. Mechanistically, lncFOXO1 suppressed the growth of breast cancer by increasing $\mathrm{FOXO1}$ transcription. Moreover, we found that 1 cFOXO1 associated with BRCA-1-associated protein 1 (BAP1) and regulates its binding at $\mathrm{FOXO1}$ promoter.

\section{Materials and methods}

Cell culture. Five breast cancer cell lines (MCF-7, BT-549, MB-231, MB-453 and MB-415) and a normal breast cell line (Hs-578Bst) were obtained from the Cell Bank of Chinese Academy of Sciences. Cells were cultured in Dulbecco's modified Eagle's medium (DMEM)/F12 (Gibco, Gaithersburg, MD, USA) supplemented with $10 \%$ fetal bovine serum (FBS; Gibco) and $1 \%$ penicillin-streptomycin (HyClone Laboratories, Inc., Logan, UT, USA). For establishing stable transfectants with lncFOXO1, cells were transfected with pCMV plasmid expressing full-length lncFOXO1.

Tissue samples. Fifty paired breast cancer and corresponding non-tumor breast tissues samples collected during breast cancer surgery were obtained from archived samples of the Tissue Bank at Cangzhou Central Hospital of Hebei Medical 
University. All the patients gave informed consent for their tissues to be used for this research. Recommendations of the Declaration of Helsinki for biomedical research involving human subjects were also followed. Ethics approval for the study was obtained from the Ethics Committee of Cangzhou Central Hospital.

Cell viability detection. Cells/well $\left(2 \times 10^{3}\right)$ were seeded in the 96-well plate and incubated for different time-points, respectively. Cell viability was measured with a Cell Counting kit-8 (CCK-8; Beyotime Institute of Biotechnology, Shanghai, China) following the manufacturer's instructions. Absorbance was measured at $450 \mathrm{~nm}$ using ELx800 reader (Bio-Tek Instruments, Inc., Winooski, VT, USA).

Colony formation assay. Cells $\left(2 \times 10^{3}\right)$ were seeded into a 6 -well plate and cultured for 1 week at $37^{\circ} \mathrm{C}$. Cells were fixed with methanol and then stained with $0.005 \%$ crystal violet. Colonies were then counted.

Western blot analysis. The cells were lysed with RIPA buffer (Beyotime Institute of Biothechnology). Protein lysates were separated by SDS-PAGE and then transferred onto PVDF membranes. The membranes were blocked and incubated with anti-FOXO1 (Abcam, Cambridge, MA, USA), or BAP1 (Abcam) and GAPDH antibody (Santa Cruz Biotechnology, Santa Cruz, CA, USA) at $4^{\circ} \mathrm{C}$ overnight. After being washed, the membranes were incubated with HRP-conjugated anti-IgG. Signal was detected by an ECL system (Amersham Pharmacia Biotech, Inc., Piscataway, NJ, USA).

Microarray analysis. Briefly, samples (6 paired breast cancer and corresponding non-tumor breast tissues) were used to synthesize double-stranded complementary DNA (cDNA), and double-stranded cDNA was labeled and hybridized to the $12 \times 135 \mathrm{~K}$ lncRNA Expression Microarray (Arraystar, Rockville, MD, USA). After hybridization and washing, processed slides were scanned with the Axon GenePix 4000B microarray scanner (Molecular Devices, Sunnyvale, CA, USA). Raw data were extracted as pair files using NimbleScan software (Roche NimbleGen, Inc., Madison, WI, USA). NimbleScan software implementation of RMA offers quantile normalization and background correction. Differentially expressed genes were identified through the random variance model. P-values were calculated using the paired $t$-test. The threshold set for upregulated and downregulated genes was a fold change $\geq 2.0$ and a $\mathrm{P}<0.05$. Hierarchical clustering was performed based on differentially expressed mRNAs and IncRNAs using Cluster TreeView software from Stanford University (Palo Alto, CA, USA).

Quantitative real-time PCR ( $q P C R)$. Total RNA was isolated using the RNeasy Mini kit (Qiagen) according to the manufacturer's instructions. Total RNA $(1 \mu \mathrm{g})$ was reversely transcribed to cDNA by using EasyScript One-Step gDNA Removal and cDNA Synthesis (TransGen Biotech, Inc., Beijing, China). qPCR was performed on the StepOnePlus Detection System (Applied Biosystems, Foster City, CA, USA). Relative expression levels were calculated as ratios normalized against those of GAPDH. Comparative quantifi-
Table I. The primers for $\mathrm{qPCR}$.

Gene symbol Sequences

GAPDH Forward: GATTCCACCCATGGCAAATTC Reverse: CTGGAAGATGGTGATGGGATT

FOXO1 Forward: GATCTACGAGTGGATGGTCAAG Reverse: CTTGCTGTGTAGGGACAGATTA

IncFOXO1 Forward: CGATGTGCTGGAGTGTATGT Reverse: GCAGGATGGCACTACTGATAA

ChIP-FOXO1 Forward: GCGGCAGATCCCGTAAGT Reverse: ACGGAAGGACGGACGGA

cation was determined using the $2^{-\Delta \Delta \mathrm{Ct}}$ method. Primers can be found in Table I.

Isolation of cytoplasmic and nuclear RNA. Cytoplasmic and nuclear RNA was isolated and purified using the Cytoplasmic and Nuclear RNA Purification kit (Norgen, Inc., Belmont, CA, USA) according to its manual.

$5^{\prime}$ and 3' Rapid amplification of cDNA ends (RACE). We used the 5'-RACE and 3'-RACE analyses to determine the transcriptional initiation and termination sites of lncRNAATB and lncRNA-508851 using a SMARTer ${ }^{\mathrm{TM}}$ RACE cDNA Amplification kit (Clontech Laboratories, Inc., Palo Alto, CA, USA) according to the manufacturer's instructions.

Cell cycle assay. The cell cycle was analyzed using an In Situ Cell Proliferation kit FLUOS (Roche Diagnostics) according to the manufacturer's instructions. The cell cycle distribution was analyzed by flow cytometry. The data were analyzed by FlowJo software (Tree Star, Inc., Ashland, OR, USA).

Apoptosis assay. Cells were stained with fluorescein isothiocyanate-conjugated Annexin V and 7-AAD (Apoptosis detection kit; Nanjing KeyGen Biotech, Co., Ltd., Nanjing, China) according to the manufacturer's instruction. Cells were analyzed with flow cytometer, and the data were studied using FlowJo software (Tree Star).

Chromatin immunoprecipitation (ChIP). ChIP assay was performed using the HiSense EZ-Magna ChIP ${ }^{\mathrm{TM}}$ Chromatin Immunoprecipitation kit (Millipore, Billerica, CA, USA), according to the manual. Briefly, crosslinked chromatin was sonicated into 200 to $1000 \mathrm{bp}$ fragments. The chromatin was immunoprecipitated using anti-BAP1 (Abcam) and antiubH2AK119 (Abcam). Normal rabbit immunoglobulin G (IgG) was used as a negative control. Quantitative PCR was conducted using SYBR-Green Mix (Takara Bio, Shiga, Japan). Primer sequences are listed in Table I.

RNA pull-down and mass spectrometry assay. RNA pulldown was performed as previously described (7). In vitro biotin-labeled RNAs (lncFOXO1 and its antisense RNA) were transcribed with the biotin RNA labeling mix (Roche) and T7 
A

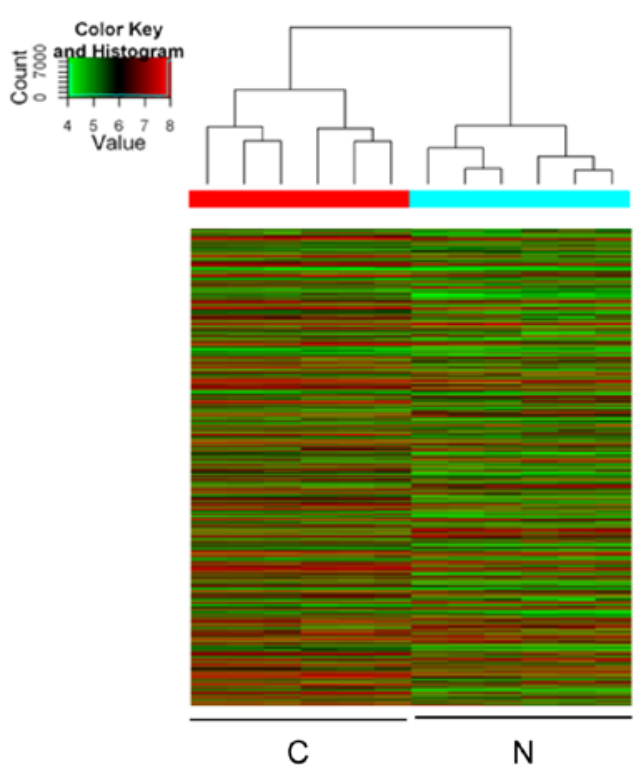

B 등
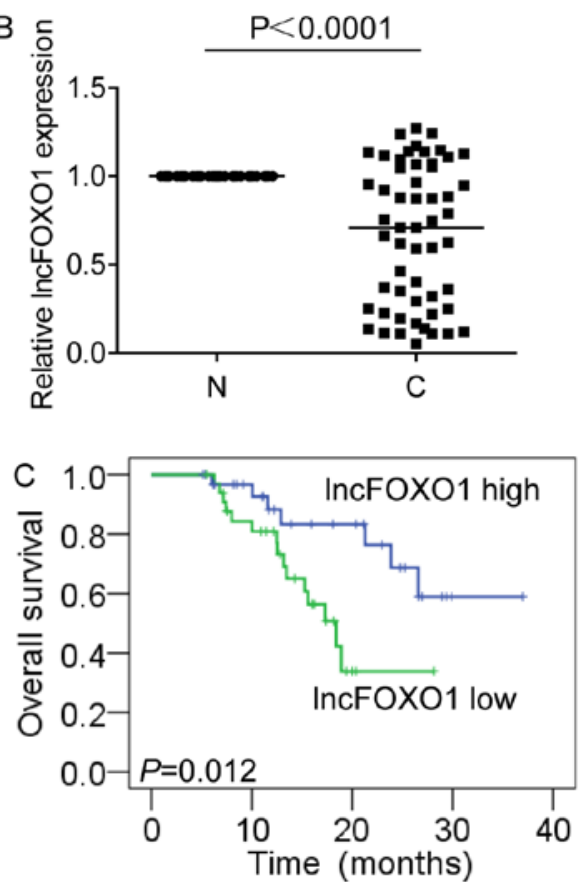

Figure 1. Downregulation of lncFOXO1 expression in human breast cancer tissues. (A) Hierarchical clustering analysis of the differentially expressed lncRNA (fold chage $>2, \mathrm{P}<0.05$ ) between tumor tissues and corresponding non-tumor tissues. (B) The relative expression of lncFOXO1 was detected by $\mathrm{qPCR}$ in 50 paired breast cancer and corresponding non-tumor breast tissues. (C) Kaplan-Meier survival curve and log-rank test were used to evaluate the association of lncFOXO1 expression level with overall survival rate. Patients were segregated into lncFOXO1-high group and lncFOXO1-low according to the median of lncFOXO1 expression in HCC.

RNA polymerase (Roche) treated with RNase-free DNase I (Promega, Madison, WI, USA) and purified with RNeasy Mini kit (Qiagen). Biotinylated RNA was incubated with nuclear extracts of breast cancer cells and pull-down proteins were run on SDS-PAGE gels. Mass spectrometry followed.

RNA-FISH. Fluorescence-conjugated lncFOXO1 probes were used for RNA-FISH. RNA-FISH was performed by using RNA-FISH kit (Wuhan Boster Biological Technology, Ltd., Wuhan, China) according to the standard protocol. Cells were observed with a FV1000 confocal laser microscope (Olympus).

Statistical analysis. All the statistical analyses were performed using SPSS software. For comparisons, two-tailed Student's t-tests were performed. Survival curve was evaluated using the Kaplan-Meier method and the differences were assessed using the log-rank test.

\section{Results}

Downregulation of IncFOXO1 expression in human breast cancer tissues. Firstly, the Agilent G3 Human GE Microarray (8x60 K) was used to analyze lncRNA expression profiles in 6 paired breast cancer tissues and corresponding non-tumor tissues. Fold change $>2.0$ and $\mathrm{P}<0.05$ between tumor tissues and corresponding non-tumor tissues were set as the criteria in filtering differently expressed IncRNAs. Results of unsupervised hierarchical clustering analysis on the significantly dysregulated lncRNAs are shown in Fig. 1A.

A previous study showed that lncRNAs act in cis to regulate expression of neighboring genes or in trans (8).
Therefore, we concentrated on intergenic lncRNAs which were aberrantly expressed in breast cancer tissues and located in the nearby coding genes related to breast development. Among these highly expressed intergenic lncRNAs, we focused on an uncharacterized lncRNA, termed 1ncFOXO1 (gene symbol RP11-172E9.2). IncFOXO1 is one of the most decreased lncRNAs in breast cancer, residing on chromosome 13 upstream of FOXOl gene. We next examined a cohort of 50 paired breast cancer and corresponding non-tumor breast tissues. We observed that lncFOXO1 was significantly decreased in breast cancer tissues (Fig. 1B). Furthermore, we found that patients with high lncFOXO1 expression in breast cancer had a better prognosis than those with low lncFOXO1 expression (Fig. 1C). Taken together, these data suggest an important role for lncFOXO1 in breast cancer.

Characterization of lncFOXO1. A total length of $683 \mathrm{nt}$ of 1ncFOXO1 transcript was determined by a RACE assay (Fig. 2A). Next, we determined the cellular location of lncFOXO1. RNA-FISH and cellular fractionation assays showed that IncFOXO1 was mainly localized in the nuclei of breast cancer cells (Fig. 2B and C).

Overexpression of lncFOXO1 inhibits cell proliferation, colony formation, and induces G0/G1 cell cycle arrest and apoptosis in breast cancer cells. Next, we determined the biological function of lncFOXO1 in breast cancer cells. Firstly, an analysis of 1ncFOXO1 expression was carried out among 5 different breast cancer cell lines (MCF-7, BT-549, MB-231, MB-453 and MB-415) and a normal breast cell line (Hs-578Bst). We noted that lncFOXO1 was obviously down- 
A 5' RACE

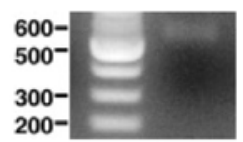

3' RACE

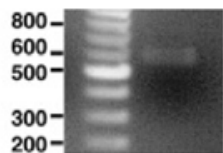

DAPI+ IncFOXO1 $\begin{gathered}\text { DAPI+ } \\ \text { IncFOXO1 }\end{gathered}$

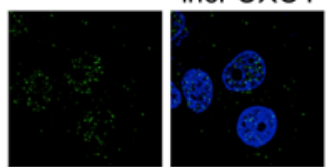

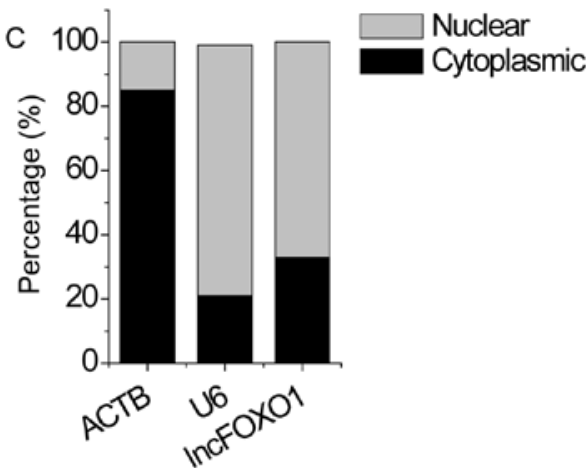

Figure 2. Characterization of IncFOXO1. (A) A representative image of PCR products from the 5'-RACE and 3'-RACE procedure. The major PCR product is marked by an arrow. (B) lncFOXO1 intracellular localization was visualized in BT-549 cells by RNA-FISH assays. (C) Fractionation of BT-549 cells followed by qPCR.
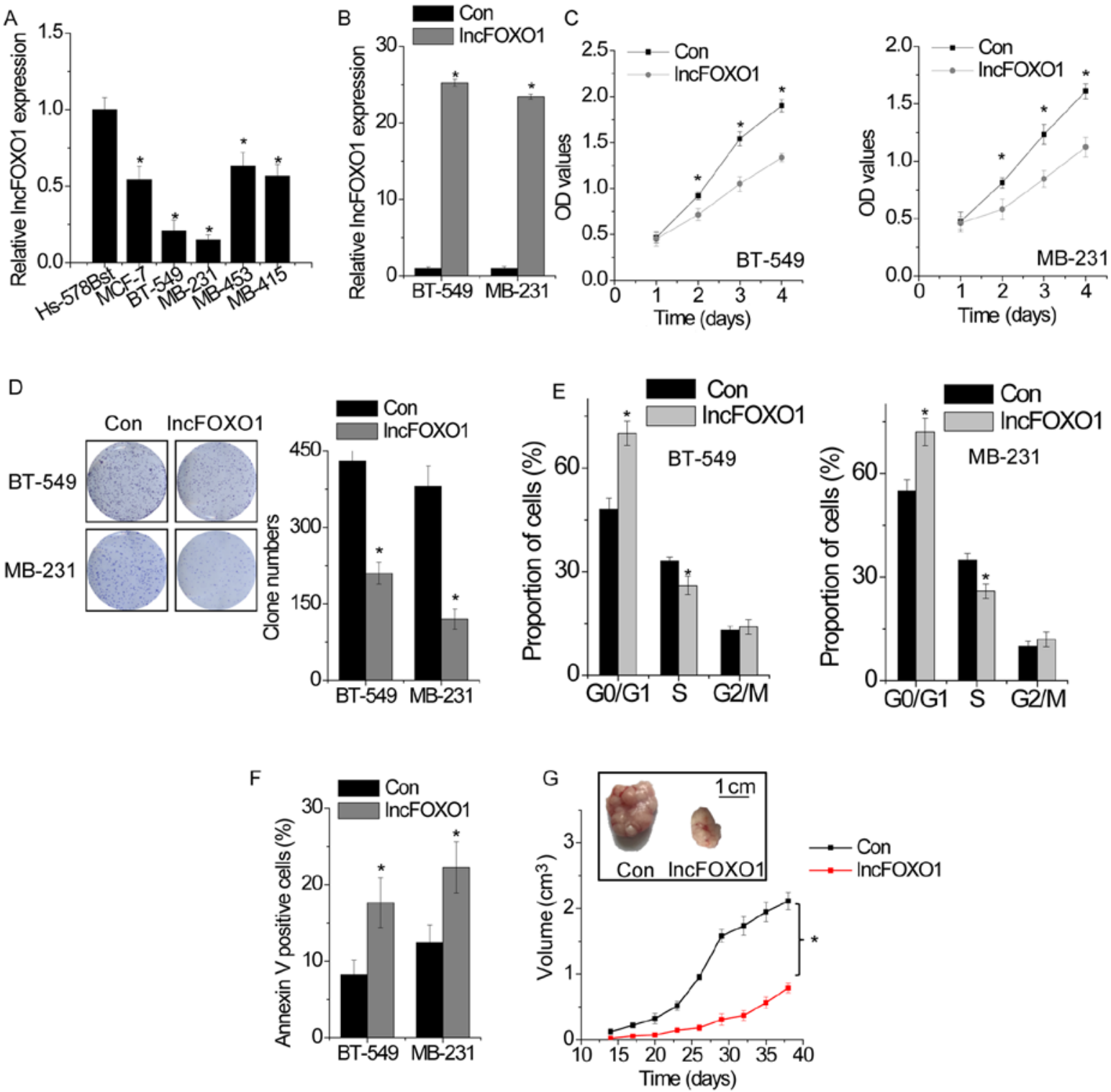

Figure 3. Overexpression of 1ncFOXO1 inhibits cell proliferation, colony formation, and induces G0/G1 cell cycle arrest and apoptosis in breast cancer cells (A) lncFOXO1 expression was determined by qPCR in normal breast cells (Hs-578Bst) and 5 different breast cancer cell lines. (B) The relative expression of lncFOXO1 in control and lncFOXO1 overexpressed cells. (C) The cell proliferation was detected by CCK-8 assay in control and lncFOXO1 overexpressed cells (D) The clone formation was detected in control and lncFOXO1 overexpressed cells. (E) The cell cycle distribution was determined in control and lncFOXO1 overexpressed cells by flow cytometry. (F) Apoptosis was determined in control and lncFOXO1 overexpressed cells by flow cytometry. Cells positive for Annexin V staining were counted as apoptotic cells, and the percentage of apoptotic cells is shown. (G) Effects of lncFOXO1 overexpression on tumor growth in vivo. Tumor growth curves measured after injection of BT-549 cells expressing control and lncFOXO1. Data are shown as mean $\pm \mathrm{SD}$. ${ }^{*} \mathrm{P}<0.05$. 
A

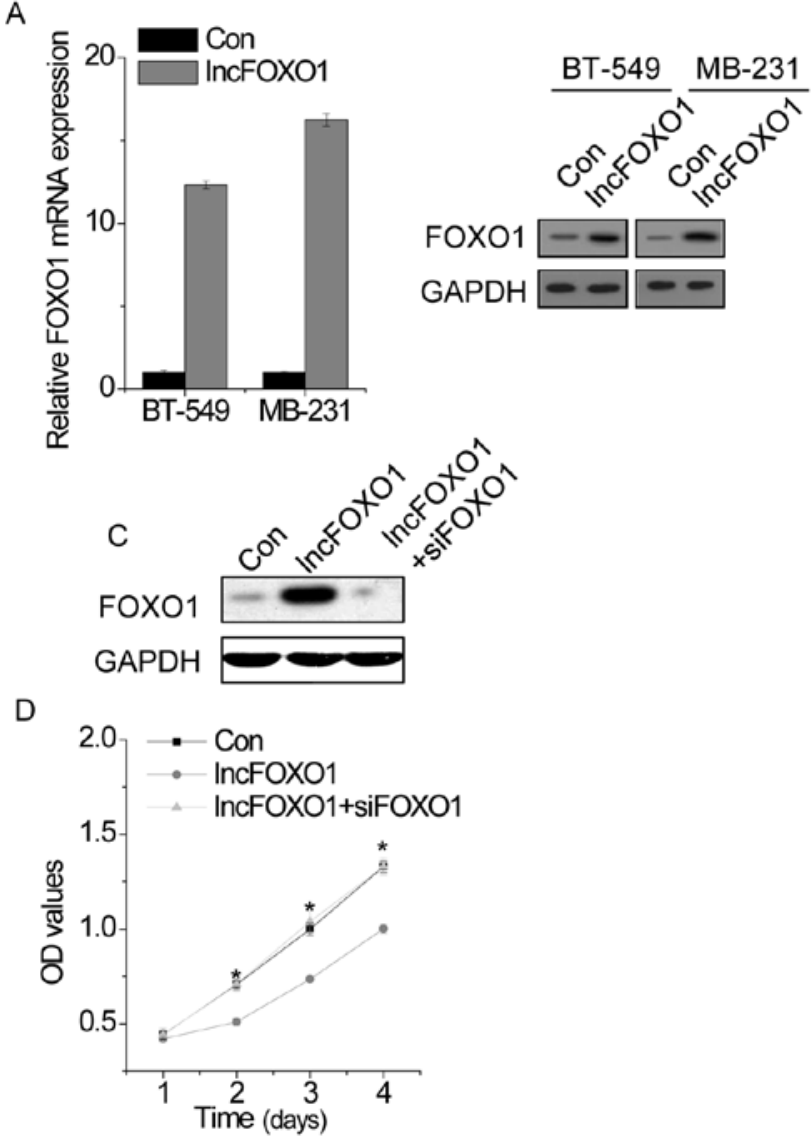

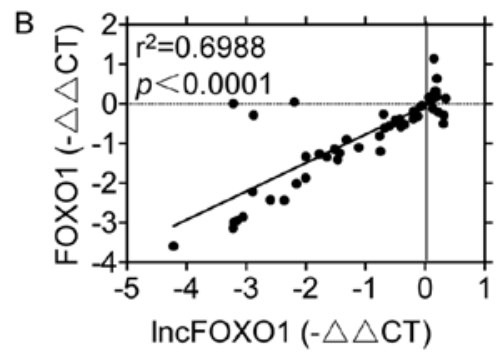

E Con IncFOXO1 IncFOXO1

E
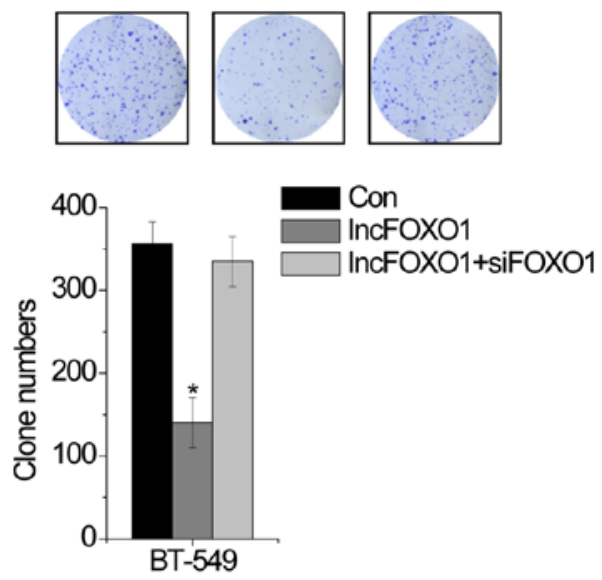

Figure 4. IncFOXO1 triggers FOXO1 expression. (A) The mRNA and protein level of FOXO1 was analyzed by qPCR and western blot analysis in control and lncFOXO1 overexpressed cells, respectively. (B) The correlation between lncFOXO1 and FOXO1 mRNA level was measured in 50 paired breast cancer and corresponding non-tumor breast tissues (Pearson correlation analysis). (C) The lncFOXO1 overexpressed cells was transfected with FOXO1 siRNA. (D) The cell proliferation was determined in lncFOXO1 overexpressed cells transiently transfected with FOXO1 siRNA. (E) The colony formation was performed in lncFOXO1 overexpressed cells transiently transfected with FOXO1 siRNA. Data are shown as mean \pm SD. ${ }^{*} \mathrm{P}<0.05$.

regulated in 5 breast cancer cell lines compared to that of Hs-578Bst cells, especially in BT-549 and MB-231 cell lines (Fig. 3A). Thus, BT-549 and MB-231 cell lines were selected as breast cancer cells in the following studies.

Then, we constructed stable cells with lncFOXO1 overexpression (Fig. 3B). To detect the effects of 1ncFOXO1 overexpression on cell proliferation, CCK-8 assay was performed. Compared with empty vector transfected cells, a significant decrease of cell viability was observed in BT-549 and MB-231 cells (Fig. 3C). To further confirm the inhibitory effect of 1ncFOXO1 overexpression on the proliferation of breast cancer cells, colony formation assay was performed. As shown in Fig. 3D, the colony numbers of BT-549 and MB-231 cells with lncFOXO1 overexpression were significantly lower than that of control cells, indicating that lncFOXO1 inhibited in vitro proliferation of breast cancer cells.

We further analyzed the cell cycle distribution and apoptosis using flow cytometry in BT-549 and MB-231 cells with lncFOXO1 overexpression. Compared with the control cells, lncFOXO1-overexpressed cells showed cell cycle arrest in G0/G1 phase (Fig. 3E). In addition, lncFOXO1 overexpression significantly promoted cell apoptosis in both BT-549 and MB-231 cells (Fig. 3F). The results showed that IncFOXO1 induces G0/G1 cell cycle arrest and apoptosis in breast cancer cells.
To assess the influence of lncFOXO1 overexpression on the in vivo growth of breast cancer cells, we applied a xenograft model in which the control and lncFOXO1 overexpressed BT-549 cells were subcutaneously injected into the flank of athymic nude mice. Our results showed that the growth of tumors from lncFOXO1-overexpression xenografts was significantly inhibited, compared with that of tumors formed from control xenografts (Fig. 3G). Taken together, these results suggest that lncFOXO1 inhibited both in vitro and in vivo growth of breast cancer cells.

lncFOXO1 triggers FOXO1 expression. Next, we explored the molecular mechanisms by which lncFOXO1 suppressed the proliferation of breast cancer cells. Firstly, we detected the neighboring gene $F O X O 1$ expression in control and lncFOXO1-overexpressed cells. Notably, lncFOXO1 overexpression significantly increased both mRNA and protein level of FOXO1 expression (Fig. 4A). We detected the expression levels of 1ncFOXO1 and FOXO1 in breast cancer samples by qPCR. We noticed that 1 cFOXO1 expression was positively correlated with the expression of FOXO1 $\left(r^{2}=0.6988\right.$, $\mathrm{P}<0.0001$; Fig. 4B). To determine whether lncFOXO1 functions upstream of FOXO1 in the regulation of cell proliferation in breast cancer, we silenced FOXO1 expression in lncFOXO1overexpressed breast cancer cells (Fig. 4C). Notably, silence 

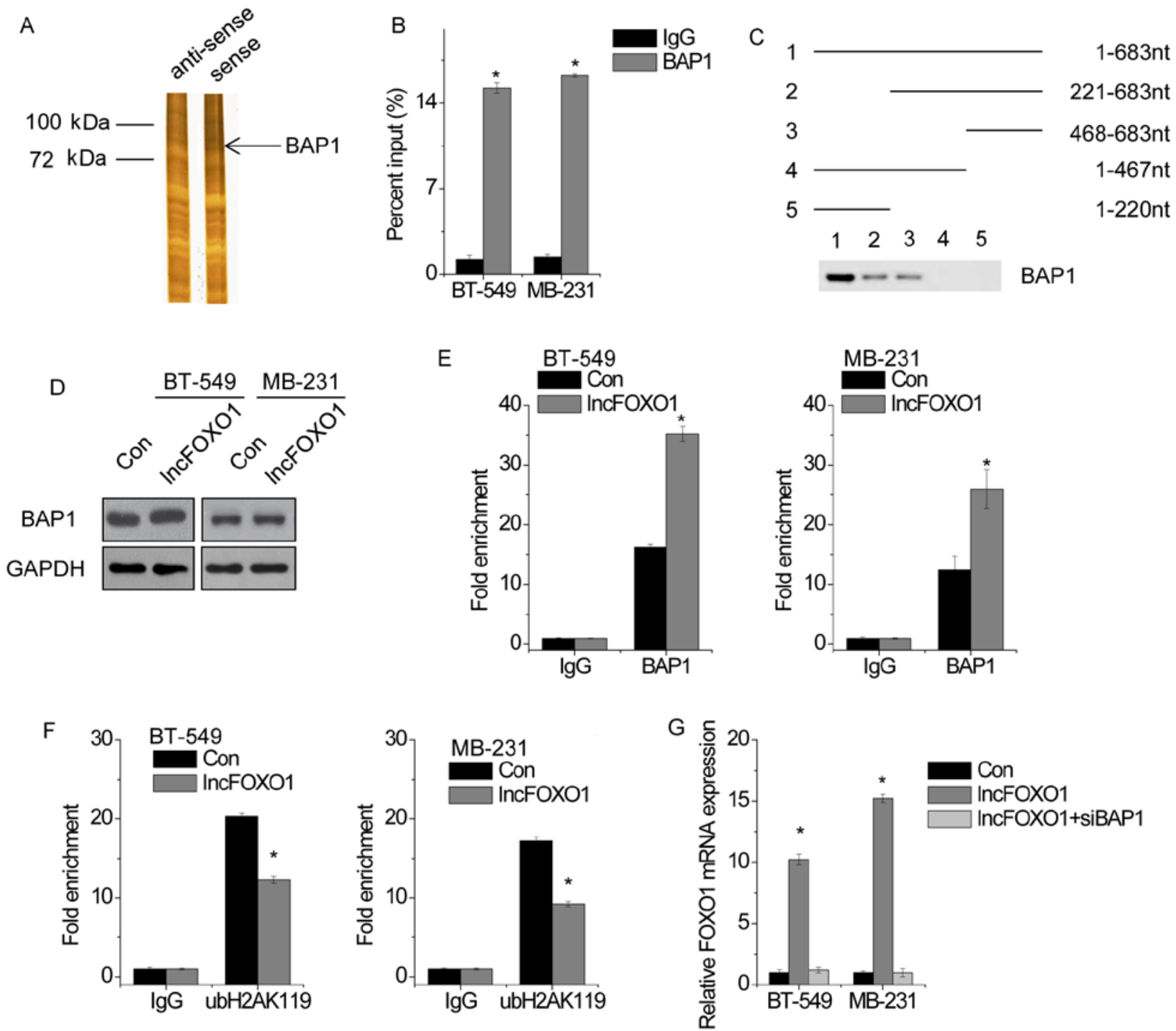

Figure 5. lncFOXO1 recruits the BAP1. (A) Biotin-RNA pull-downs were performed with nuclear extracts of oncosphere cells using full-length lncFOXO1 transcript (sense) and antisense. This was followed by mass spectrometry. (B) The interaction of lncFOXO1 with BAP1 was verified by an RNA immunoprecipitation (RIP) assay. (C) Mapping analysis of BAP1-binding domains of lncFOXO1. Shown are the following: schematic diagram of lncFOXO1 full-length and truncated fragments (top panel) and western blot analysis of BAP1 in RNA pull-down samples by different lncFOXO1 fragments (bottom panel). (D) The protein level of BAP1 was analyzed by western blot analysis in control and lncFOXO1 overexpressed cells. (E) The binding level of BAP1 at FOXO1 promoter region was determined by ChIP assay and followed by qPCR in control and lncFOXO1 overexpressed cells. (E) The ubH2AK119 level of FOXO1 promoter region was determined by ChIP assay and followed by qPCR in control and lncFOXO1 overexpressed cells. (G) BAP1 deletion significantly abolished the upregulation of FOXO1 induced by 1 ncFOXO1 overexpression. Data are shown as mean $\pm \mathrm{SD}$. ${ }^{*} \mathrm{P}<0.05$.

of FOXO1 abolished the growth suppression induced by lncFOXO1 overexpression (Fig. 4D and E). These data demonstrated that lncFOXO1 suppressed cell proliferation of breast cancer cells through upregulation of FOXO1.

BAP1 is recruited by IncFOXO1. Finally, we explored the regulatory mechanism of FOXO1 expression mediated by lncFOXO1. IncRNAs are considered to exert their functions through RNA-interacting proteins that regulate gene expression by various mechanisms. Therefore, we performed an RNA pull-down assay with biotin-labeled lncFOXO1 and followed by mass spectrum to search for potential lncFOXO1-associated proteins. The deubiquitinating enzyme BRCA-1-associated protein 1 (BAP1) were identified to potentially interact with lncFOXO1 in breast cancer cells (Fig. 5A). The interaction of lncFOXO1 with BAP1 was further validated by RNA immu- noprecipitation (RIP) (Fig. 5B). We next constructed a series of lncFOXO1 truncations to map its binding fragment with BAP1. We found that the 3'-end fragment of lncFOXO1 (468 to $683 \mathrm{nt}$ ) was essential to bind BAP1 (Fig. 5C).

lncFOXO1 overexpression did not influence protein level of BAP1 (Fig. 5D), suggesting that lncFOXO1 was not involved in the post-translational regulation of the BAP1. Given that the BAP1 regulates gene transcription via binding to promoter region and deubiquitinate mono-ubiquitinated $\mathrm{H} 2 \mathrm{~A}$ at K119 (ubH2AK119), we then detected whether IncFOXO1 influenced BAP1 occupancy and the level of ubH2AK119 of the FOXO1 promoter by performing ChIP assays. We found that lncFOXO1 overexpression increased the binding of BAP1 to $F O X O 1$ promoter (Fig. 5E), and decreased the level of ubH2AK119 across $F O X O 1$ promoter region (Fig. 5F). In addition, BAP1 deletion significantly abolished the upregulation 
of FOXO1 induced by lncFOXO1 overexpression (Fig. 5G). Taken together, our data demonstrated that lncFOXO1 triggers FOXO1 expression through recruitment of BAP1, leading to suppression of the proliferation of breast cancer cells.

\section{Discussion}

In the present study, we revealed the critical roles of lncFOXO1 in breast cancer proliferative phenotype and clinical prognosis, based on the following observations: i) lncFOXO1 is significantly decreased in breast cancer tissues and cell lines; ii) downregulation of lncFOXO1 expression associates with poorer overall survival; iii) overexpression of lncFOXO1 inhibits the in vitro and in vivo growth of breast cancer cells; iv) lncFOXO1 activates FOXO1 expression through recruiting BAP1 and decreasing the ubH2AK119 at $\mathrm{FOXO1}$ promoter.

Increasing evidence reports that numerous $\operatorname{lncRNAs}$ are frequently aberrantly expressed in different kinds of cancers, exhibiting regulated gene expression variously $(9,10)$. These differential expression lncRNAs are closely associated to tumorigenesis, metastasis, prognosis or diagnosis, serving as oncogenes or/and tumor suppressor genes (9). However, there are few studies on tumor suppressive lncRNA in breast cancer. In the present study, we identified a novel lncRNA that exerts tumor suppressive activity in breast cancer. We found that lncFOXO1 inhibits the growth of breast cancer cells both in vitro and in vivo. IncFOXO1 may be a potential target for therapy of breast cancer.

Notably, accumulating evidence shows that lncRNAs modulate gene expression as epigenetic modifiers. Here, for the first time, we found that lncFOXO1 activates FOXO1 expression through recruitment of BAP1. BAP1 is a critical tumor suppressor, and loss of BAP1 expression leads to a variety of cancers including pleural mesothelioma, renal cell carcinoma and bladder tumors. Decrease of BAP1 expression is significantly associated with high tumor aggressiveness and poor prognosis (11-13). BAP1 regulates the expression of target genes through association with transcription-related proteins such as ASXL1 and ASXL2. BAP1 deubiquitinates ubH2AK119 of target genes to activate their transcription (14). In the present study, we found that FOXOI also is a direct target gene of BAP1. Overexpression of IncFOXO1 increased the binding of BAP1 at $\mathrm{FOXO1}$ promoter, and decreased the level of ubH2AK119 across FOXO1 promoter. Overall, we proposed a novel model in which lncFOXO1 associates with chromatin-modifying factor BAP1 to regulate FOXO1 expression in breast cancer.

Previous studies reported that lncRNAs can act in cis to regulate expression of neighboring genes. For example, lncTCF7 recruits the SWI/SNF complex to the promoter of $T C F 7$ to regulate its expression, leading to activation of $\mathrm{Wnt}$ signaling (15). FOXO1 is considered as a tumor suppressor that inhibits cell proliferation and induce apoptosis. FOXO1 alterations have been described in a number of human cancers, such as rhabdomyosarcoma, leukemia and lymphoma (16-18). In addition, FOXO1 proteins are inactivated by major oncogenic signals such as the phosphatidylinositol-3 kinase pathway (PI3K) and MAP kinases (MAPK) or microRNAs (19-21). In this study, we revealed a new regulatory mechanism of FOXO1 expression. We found that lncFOXO1 activates the expression of neighboring gene, FOXO1, through interaction with BAP1. Understanding the precise molecular mechanisms by which IncRNAs exert their function in breast cancer will be critical for exploring these potential new strategies for early diagnosis and therapy of breast cancer.

In conclusion, IncFOXO1 suppresses the growth of breast cancer cells by recruiting BAP1 to the $\mathrm{FOXO1}$ promoter. Our findings reveal that lncRNAs may represent an additional layer of regulation of the tumor suppressor.

\section{Acknowledgements}

The present study was supported by grants from the Cangzhou Key Technology Support Program (no. 131302120).

\section{References}

1. Wilusz JE, Sunwoo H and Spector DL: Long noncoding RNAs: Functional surprises from the RNA world. Genes Dev 23: 1494-1504, 2009.

2. Geisler S and Coller J: RNA in unexpected places: Long noncoding RNA functions in diverse cellular contexts. Nat Rev Mol Cell Biol 14: 699-712, 2013.

3. Prensner JR and Chinnaiyan AM: The emergence of lncRNAs in cancer biology. Cancer Discov 1: 391-407, 2011.

4. Dieli-Conwright CM, Lee K and Kiwata JL: Reducing the risk of breast cancer recurrence: An evaluation of the effects and mechanisms of diet and exercise. Curr Breast Cancer Rep 8: 139-150, 2016.

5. Zhao Z, Li S, Song E and Liu S: The roles of ncRNAs and histone-modifiers in regulating breast cancer stem cells. Protein Cell 7: 89-99, 2016.

6. Cojocneanu Petric R, Braicu C, Raduly L, Zanoaga O, Dragos N, Monroig P, Dumitrascu D and Berindan-Neagoe I: Phytochemicals modulate carcinogenic signaling pathways in breast and hormone-related cancers. Onco Targets Ther 8: 2053-2066, 2015.

7. Hu X, Feng Y, Zhang D, Zhao SD, Hu Z, Greshock J, Zhang Y, Yang L, Zhong X, Wang LP, et al: A functional genomic approach identifies FAL1 as an oncogenic long noncoding RNA that associates with BMI1 and represses p21 expression in cancer. Cancer Cell 26: 344-357, 2014.

8. Rinn JL and Chang HY: Genome regulation by long noncoding RNAs. Annu Rev Biochem 81: 145-166, 2012.

9. Takenaka K, Chen BJ, Modesitt SC, Byrne FL, Hoehn KL and Janitz M: The emerging role of long non-coding RNAs in endometrial cancer. Cancer Genet 209: 445-455, 2016.

10. Chen QN, Wei CC, Wang ZX and Sun M: Long non-coding RNAs in anti-cancer drug resistance. Oncotarget 8: 1925-1936, 2016.

11. Jaouen A, Thivolet-Bejui F, Chalabreysse L, Piaton E, TraverseGlehen A, Isaac S, Decaussin-Petrucci M, Depaepe L, Fontaine J, Remy I, et al: BRCA1 associated protein $1(B A P 1)$ expression in pleural diffuse malignant mesothelioma: A comparative cytological and histological analyses on 50 patients. Ann Pathol 36: 111-119, 2016 (In French).

12. Minardi D, Lucarini G, Milanese G, Di Primio R, Montironi R and Muzzonigro G: Loss of nuclear BAP1 protein expression is a marker of poor prognosis in patients with clear cell renal cell carcinoma. Urol Oncol 34: 338.e11-338.e18 , 2016.

13. Nickerson ML, Dancik GM, Im KM, Edwards MG, Turan S, Brown J, Ruiz-Rodriguez C, Owens C, Costello JC, Guo G, et al: Concurrent alterations in TERT, KDM6A, and the BRCA pathway in bladder cancer. Clin Cancer Res 20: 4935-4948, 2014.

14. Sahtoe DD, van Dijk WJ, Ekkebus R, Ovaa H and Sixma TK: BAP1/ASXL1 recruitment and activation for $\mathrm{H} 2 \mathrm{~A}$ deubiquitination. Nat Commun 7: 10292, 2016.

15. Wang Y, He L, Du Y, Zhu P, Huang G, Luo J, Yan X, Ye B, Li C, Xia P, et al: The long noncoding RNA lncTCF7 promotes self-renewal of human liver cancer stem cells through activation of Wnt signaling. Cell Stem Cell 16: 413-425, 2015. 
16. Bharathy N, Suriyamurthy S, Rao VK, Ow JR, Lim HJ, Chakraborty P, Vasudevan M, Dhamne CA, Chang KT, Min VL, et al: $\mathrm{P} / \mathrm{CAF}$ mediates PAX3-FOXO1-dependent oncogenesis in alveolar rhabdomyosarcoma. J Pathol 240: 269-281, 2016.

17. Wagle M, Eiring AM, Wongchenko M, Lu S, Guan Y, Wang Y, Lackner M, Amler L, Hampton G, Deininger MW, et al: A role for FOXO1 in BCR-ABL1-independent tyrosine kinase inhibitor resistance in chronic myeloid leukemia. Leukemia 30: 1493-1501, 2016.

18. Szydlowski M, Kiliszek P, Sewastianik T, Jablonska E, Bialopiotrowicz E, Gorniak P, Polak A, Markowicz S, Nowak E, Grygorowicz MA, et al: FOXO1 activation is an effector of SYK and AKT inhibition in tonic BCR signal-dependent diffuse large B-cell lymphomas. Blood 127: 739-748, 2016.
19. Kim JH, Choi JS and Lee BH: PI3K/Akt and MAPK pathways evoke activation of FoxO transcription factor to undergo neuronal apoptosis in brain of the silkworm Bombyx mori (Lepidoptera: Bombycidae). Cell Mol Biol (Noisy-le-grand) (Suppl 58): OL1780-OL1785, 2012.

20. Lian R, Lu B, Jiao L, Li S, Wang H, Miao W and Yu W: MiR-132 plays an oncogenic role in laryngeal squamous cell carcinoma by targeting FOXO1 and activating the PI3K/AKT pathway. Eur J Pharmacol 792: 1-6, 2016.

21. Zeng YB, Liang XH, Zhang GX, Jiang N, Zhang T, Huang JY, Zhang L and Zeng XC: miRNA-135a promotes hepatocellular carcinoma cell migration and invasion by targeting forkhead box O1. Cancer Cell Int 16: 63, 2016. 